OPEN ACCESS

Edited by:

Eiman Aleem,

University of Arizona, United States

Reviewed by:

Zhaozhu Li,

The Second Affiliated Hospital of Harbin Medical University, China Alessia Peserico,

University of Teramo, Italy

${ }^{*}$ Correspondence:

Mingming Jin

asdjinmingming@126.com

Chengaing $Y$

ycq3000@126.com

Specialty section:

This article was submitted to

Cell Growth and Division,

a section of the journal

Frontiers in Cell and Developmental

Biology

Received: 17 August 2020

Accepted: 24 May 2021

Published: 27 August 2021

Citation:

Yu Y, Sun B, Wang $Z$, Yang $M$, Cui Z, Lin S, Jin M and Yi C (2021) Exosomes From M2 Macrophage

Promote Peritendinous Fibrosis

Posterior Tendon Injury via the MiR-15b-5p/FGF-1/7/9 Pathway

by Delivery of circRNA-Ep400.

Front. Cell Dev. Biol. 9:595911.

doi: 10.3389/fcell.2021.595911

\section{Exosomes From M2 Macrophage Promote Peritendinous Fibrosis Posterior Tendon Injury via the MiR-15b-5p/FGF-1/7/9 Pathway by Delivery of circRNA-Ep400}

\author{
Yinxian Yu', Binbin Sun'2, Zhuoying Wang ${ }^{1}$, Mengkai Yang ${ }^{1}$, Zhi Cui', Subin Lin ${ }^{3}$, \\ Mingming Jin ${ }^{4 *}$ and Chengqing $\mathrm{Yi}^{1 *}$
}

\begin{abstract}
1 Department of Orthopaedic Surgery, Shanghai General Hospital, Shanghai Jiao Tong University School of Medicine, Shanghai, China, ${ }^{2}$ Department of Orthopaedic Surgery, Shanghai Ninth People's Hospital, Shanghai Jiao Tong University School of Medicine, Shanghai, China, ${ }^{3}$ Department of Orthopedics, The Second Affiliated Hospital of Soochow University, Suzhou, China, ${ }^{4}$ Shanghai Key Laboratory of Molecular Imaging, Shanghai University of Medicine and Health Sciences, Shanghai, China
\end{abstract}

Achilles tendon rupture prognosis is usually unsatisfactory. After the tendon is injured, it may not function properly because of the fibrotic healing response, which restrains tendon motion. Inflammatory monocytes and tissue-resident macrophages are indispensable regulators in tissue repair, fibrosis, and regeneration. Exosomes from macrophages are crucial factors in tissue microenvironment regulation following tissue injury. This study therefore aimed to clarify the roles of macrophage exosomes in tendon injury (TI) repair. The results show that macrophages play a role after TI. M1 macrophages were increased relative to peritendinous fibrosis after TI. Highthroughput sequencing showed abnormal expression of circular RNAs (circRNAs) between exosomes from M2 and M0 macrophages. Among the abnormal expressions of circRNA, circRNA-Ep400 was significantly increased in M2 macrophage exosomes. The results also show that M2 macrophage-derived circRNA-Ep400-containing exosomes are important for promoting peritendinous fibrosis after TI. Bioinformatics and dual-luciferase reporting experiments confirmed that miR-15b-5p and fibroblast growth factor (FGF)-1/7/9 were downstream targets of circRNA-Ep400. High circRNAEp400-containing exosome treatment inhibited miR-15b-5p, but promoted FGF1/7/9 expression in both fibroblasts and tenocytes. Furthermore, high circRNA-Ep400containing exosome treatment promoted fibrosis, proliferation, and migration in both fibroblasts and tenocytes. Taken together, the results show that M2 macrophagederived circRNA-Ep400-containing exosomes promote peritendinous fibrosis after $\mathrm{TI}$ via the miR-15b-5p/FGF-1/7/9 pathway, which suggests novel therapeutics for tendon injury treatment.

Keywords: M2 macrophage, exosomes, circRNA-Ep400, tendon injury, miR-15b-5p, FGF-1/7/9

Abbreviations: circRNA, circular RNA; TI, tendon injury; miRNA, microRNA; 3'-UTR, 3'-untranslated region. 


\section{INTRODUCTION}

Tendon tears and tendinopathies are common musculoskeletal injuries, which contribute to $>30 \%$ of musculoskeletal consultations (McCormick et al., 1995). Flexor tendon injury (TI) usually occurs during lacerations, and the incidence is highest among patients 20-29 years of age, with a higher incidence in males than in females (de Jong et al., 2014). Of these injuries, tendinopathy has a huge socioeconomic burden, predicted to result in an annual medical cost and indirect lost salary expenditure of approximately $\$ 850$ billion (Wang et al., 2018). After injury, tendons usually heal through peritendinous fibrosis, without leading to regeneration of natural tendon structure, which involves adhesion between the nearby synovial sheath and tendon, resulting in impaired tendon gliding and ultimately in tissue stiffness (Wynn and Ramalingam, 2012; Zhou et al., 2018).

Tendon disorders are common and lead to significant disability, pain, healthcare costs, and lost productivity. A wide range of injury mechanisms exist leading to tendinopathy or tendon rupture (Pitt et al., 2016). Tendon healing after surgical repair generally progresses through a short inflammatory phase, which lasts about a week; followed by a proliferative phase, which lasts a few weeks; followed by a remodeling phase, which lasts many months (Zhang et al., 2015). During the inflammatory phase, vascular permeability increases and an influx of inflammatory cells enters the healing site. These cells produce a number of cytokines and growth factors that lead to recruitment and proliferation of macrophages and resident tendon fibroblasts. During the proliferative and remodeling phases of healing, fibroblasts proliferate and begin to produce, deposit, orient, and crosslink fibrillar collagens (Thomopoulos et al., 2015). Previous studies have found the potential of macrophages to convert into collagen-producing fibroblast-like cells, in the absence of any other accessory cells. This macrophage to fibroblast-like transition might augment fibrosis by collagen deposition (Haider et al., 2019).

Recent evidence suggests that modulation of inflammation in the early stages following tendon repair may lead to improved healing (Hays et al., 2008). Macrophages play essential roles in both promoting and resolving inflammation and in both facilitating and moderating tissue repair. In an injury setting, M1 cells predominate early, whereas M2 macrophages accumulate later (Lichtnekert et al., 2013). Increasing numbers of studies have found that macrophages affect the organizational microenvironment by secretion of exosomes and transmission of molecules with biological information (Momen-Heravi et al., 2014).

Exosomes are small endocytic origin membrane vesicles generated by the majority of cells in culture. Exosomes are the main extracellular signaling pathways, which are implicated in various traits and are involved in different physiological processes (Zhang et al., 2015; Pitt et al., 2016; Thomou et al., 2017; Ying et al., 2017). They originate from fusion interactions among cells and carriers of genetic information, which function in repairing inflammation and tissue when shuttled by exosomes (Bartel, 2004; Wang C. et al., 2017; Ying et al., 2017). An increasing number of studies have reported that macrophages function in tissue repair, regeneration, and fibrosis (Wynn and Vannella, 2016). Persistent tilt polarization toward M2 macrophages correlates with fibrosis and the epithelial-mesenchymal transition (Yao et al., 2016; Wang et al., 2019). In addition, whether exosomes from M2 macrophages have an inductive effect during peritendinous fibrosis after TI is unknown. This study therefore aimed to characterize the therapeutic mechanism of M2 macrophage exosomes after TI, and to then identify the therapeutic mechanism of how M2 macrophage exosomes promote peritendinous fibrosis posterior TI.

\section{MATERIALS AND METHODS}

\section{Animals and Macrophage Depletion}

The Animal Research Committee in Shanghai Jiao Tong University Affiliated Shanghai First People's Hospital approved the animal protocols. We performed all procedures following NIH Guidelines regarding Laboratory Animal Care and Use.

We used phosphate-buffered saline (PBS)-Lipo or Clo-Lipo ${ }^{1}$ following a published protocol (Bartel, 2004). In brief, 2 days before tendon surgery, we administered six- to 8-week-old male C57BL/6 mice $1.33 \mathrm{~mL} / \mathrm{kg}$ of PBS-Lipo or Clo-Lipo by intraperitoneal injection, and injected another $1.33 \mathrm{~mL} / \mathrm{kg}$ of Clo-Lipo directly into the tendon during surgery. The animals received the same weekly dosage $(1.33 \mathrm{~mL} / \mathrm{kg})$ of injections during the procedure. Each group was comprised of six mice.

\section{Flexor Tendon Surgery}

The flexor digitorum longus (FDL) tendons in the mouse right hind paw were fully transected and repaired (Cui et al., 2019). In summary, we anesthetized animals by an injection of $4 \mathrm{mg} / \mathrm{kg}$ xylazine and $60 \mathrm{mg} / \mathrm{kg}$ ketamine. After surgical site preparation, we performed surgery through the FDL in the transverse plane at the calf myotendinous junction to ensure that the repair site would not be affected by high strain. We closed the skin using 60 nylon running sutures (Ethicon, Edinburgh, United Kingdom). Afterward, we made a $2 \mathrm{~cm}$ incision on the posterior hind paw surface, and retracted soft tissue to detect the FDL. We fully transected the FDL using microscissors and then used 8-0 prolene sutures (Ethicon) in a modified Kessler pattern for repair. We then closed the skin using 6-0 nylon running sutures. The sham surgery was conducted in the control group using an identical exposure protocol and anesthesia, but the isolated FDL tendon was not damaged. We then returned the mice to their cages and allowed them to move freely and bear weight after recovery from anesthesia. After allowing FDL tendon repair for 3 weeks, the mice were sacrificed by cervical dislocation and used for subsequent analyses. Each group was comprised of six mice.

\section{Mechanical Testing}

To test the mechanical properties of the healing tendon after macrophage treatment, day 14 tendons were mechanically tested. Achilles tendons were carefully dissected and surrounding tissue excised to keep the calcaneal insertion site intact. Tendons

\footnotetext{
${ }^{1}$ http://www.clodronateliposomes.org
} 
remained hydrated using PBS. Tendon length, width, and thickness were measured using a dissecting microscope and digital calipers during the pre-load. Width and thickness measurements were obtained at the injury site. The cross sectional area (assumed to be an ellipse) was then estimated. Tendons were tested in a custom-designed load frame, which gripped and loaded the tendons along their longitudinal axis. The calcaneus was trimmed and press-fit into a custom bone grip. The soft tissue end of the specimens was fixed to strips of Tyvek (McMaster-Carr, Elmhurst, IL, United States) with adhesive (super glue; Ace Hardware, Oak Brook, IL, United States), which were held between two plates to form the soft-tissue grip. Dimensional measurements for the tendons were recorded at preload. Mechanical testing was performed at room temperature. A low preload of $0.1 \mathrm{~N}$ was applied in order to obtain a uniform zero point prior to preconditioning $(20$ cycles at $0.5 \mathrm{~Hz})$ to $0.5 \%$. Pull-to-failure testing was performed on tendons at a rate of $3.33 \mathrm{~mm} / \mathrm{s}$. Force and displacement data from the test system were recorded at $10 \mathrm{~Hz}$. Failure force was the highest load prior to failure of the tendon, and Lagrangian stress was calculated by dividing the failure force by the initial cross-sectional area of the tendon. Stiffness was calculated by determining the slope of the most linear portion of the load-displacement curve. Young's modulus was calculated using the slope of the linear portion of the stress-strain curve.

\section{Histological Evaluation}

After euthanizing the mice, skin and excess soft tissue were removed at the mid tibia, while skin on the foot sole remained intact and did not affect the repair site. The samples were then fixed for 1 day using 4\% paraformaldehyde (PFA) in PBS. After dehydration in a series of ethanol gradients and embedding in paraffin, we cut $4 \mu \mathrm{m}$ sagittal slices, which were stained with Masson's trichrome, F4/80, and for inducible nitric oxide synthase (iNOS).

\section{Cell Culture}

We purchased the NIH 3T3 fibroblast cell line from the Cell Bank of the Type Culture Collection in the Chinese Academy of Sciences (Shanghai Institute of Cell Biology, Shanghai, China) and cultured them in Dulbecco's Modified Eagle's Medium (DMEM; Gibco, Gaithersburg, MD, United States) containing 10\% fetal bovine serum (FBS; Gibco).

We harvested tenocytes from mouse superficial flexor tendons (Thomopoulos et al., 2015). In brief, we digested tendon pieces with $0.15 \%$ collagenase NB4 (SERVA Electrophoresis, Heidelberg, Germany) for $2 \mathrm{~h}$ at $37^{\circ} \mathrm{C}$. We then filtered the mixture through cell mesh $(60 \mu \mathrm{m})$ (Corning, Corning, NY, United States). After centrifugation at $300 \times g$ for $5 \mathrm{~min}$, we resuspended the cell pellet in DMEM containing 10\% FBS and 1\% penicillin-streptomycin.

We separated bone marrow-derived macrophages (BMDMs; M0 macrophages) from adult mice femurs and tibias. We flushed tibias, and seeded bone marrow cells into $10 \mathrm{~cm}^{2}$ tissue culture plates, which were cultured in alpha minimum essential medium (alpha MEM; Gibco) with 10\% FBS and 1\% penicillinstreptomycin at $37^{\circ} \mathrm{C}$, supplemented with $10^{4} \mathrm{U} / \mathrm{mL}$ macrophage colony-stimulating factor for 1 day. We cultured BMDMs in
alpha-MEM with $10 \%$ exosome-free FBS to generate exosomes. For M2 macrophage induction, $20 \mathrm{ng} / \mathrm{mL}$ IL-13 and IL-4 were used for 1 day to treat M0 macrophages as previously described (Thomou et al., 2017).

\section{Exosome Purification, Labeling, and Characterization}

After 3 days of macrophage culture, we removed dead cells and debris in the medium by centrifugation at $1,000 \times g$ for $10 \mathrm{~min}$ and filtered the samples using a $0.2 \mu \mathrm{m}$ filter. We then subjected the medium to ultracentrifugation at $100,000 \times g$ for $4-6 \mathrm{~h}$ at $4^{\circ} \mathrm{C}$. After washing with PBS $(100,000 \times g$ for $20 \mathrm{~min})$, we resuspended the exosome-containing pellet in PBS. For electron microscopy, we fixed the exosomes with $2 \%$ PFA and mounted them on a Formvar- and carbon-coated copper mesh grid. The exosomes were placed the grids on $2 \%$ gelatin for $20 \mathrm{~min}$ at $37^{\circ} \mathrm{C}$, rinsed with $0.15 \mathrm{M}$ glycine and PBS and blocked using $1 \%$ cold water fish skin gelatin. The grids were then viewed using transmission electron microscopy.

To track the exosomes, we labeled exosomes with fluorescent dye using a PKH26 fluorescent cell linker kit (Sigma-Aldrich, St. Louis, MO, United States), following standard procedures. After labeling, the PKH26-exosomes were rinsed with PBS, concentrated using ultracentrifugation $(100,000 \times g$ for $20 \mathrm{~min})$ at $4^{\circ} \mathrm{C}$ and resuspended the in PBS.

\section{High-Throughput and Strand-Specific RNA-Seq Library Construction}

Total RNA was extracted from exosomes isolated from M0 and M2 macrophages using TRIzol reagent (Invitrogen, Carlsbad, CA, United States). We obtained approximately $3 \mu \mathrm{g}$ total RNA from every specimen using the VAHTS Total RNA-seq (H/M/R) Library Prep Kit from Illumina (Vazyme Biotech, Nanjing, China) to eliminate ribosomal RNA, but retained other RNAs like ncRNA and mRNA. We treated the purified RNA with RNase R ( $40 \mathrm{U}$, for $3 \mathrm{~h}$ at $37^{\circ} \mathrm{C}$ ) (Epicenter), which was followed by TRIzol purification. We constructed a RNA-seq library using the KAPA Stranded RNA-Seq Library Prep Kit (Roche, Basel, Switzerland) and deep sequencing with a HiSeq 4000 (Illumina, San Diego, CA, United States) conducted by Aksomics (Shanghai, China).

\section{Bioinformatic Analysis}

We predicted circRNA/miRNA target genes using Circular RNA Interactome. We predicted interactions between miR-15b-5p and FGF using ${ }^{2}$.

\section{Fluorescence in situ Hybridization (FISH)}

We obtained specific probes to circRNA-Ep400 (Dig-5' ACAGGTGGACCCAGAACCAGCCAG-3'-Dig) from Geneseed Biotech (Guangzhou, China). FITC-conjugated anti-biotin antibodies were also purchased from Jackson ImmunoResearch (West Grove, PA, United States). We counterstained the nuclei with 4,6-diamidino-2-phenylindole (DAPI). Finally, we obtained images using an LSM 700 confocal microscope (Carl Zeiss, Oberkochen, Germany).

\footnotetext{
${ }^{2}$ http://www.targetscan.org/
} 


\section{Total RNA Isolation and RT-qPCR}

Total RNA was isolated from cells and tissues utilizing TRIzol reagent (Invitrogen), using standard procedures. We spectrophotometrically examined the concentration and purity of RNA samples by detecting absorbance at 230, 260, and $280 \mathrm{~nm}$ using a NanoDrop ND-1000 (Thermo Fisher Scientific, Wilmington, DE, United States). Specifically, we assumed that OD260/OD280 ratios of 1.8-2.1 were acceptable, and that OD260/OD230 ratios greater than 1.8 were acceptable.

We reverse transcribed total RNA previously detected using RT-qPCR. The primers specific for circRNA-Ep 400 and miR-15b-5p, as well as FGF1/7/9 were obtained from GenePharma (Shanghai, China). RT-qPCR was performed using an AB7300 thermo-recycler (Applied Biosystems, Carlsbad, CA, United States) leveraging primers and the TaqMan Universal PCR Master Mix. The GAPDH gene was used as a reference for mRNAs and circRNAs. U6 was used as an internal control for the miRNA expression scale. Gene expression was quantified by $2^{-\Delta \Delta C t}$. Primers utilized to assay circRNA-Ep400 expression included forward, 5'-GGACTTCGGAGAGCTTC- 3 ' and reverse, $5^{\prime}$-CCGTCTCCCTGTGGTCGTC- $3^{\prime}$. The miR-15b-5p primer was, $5^{\prime}$-TAGCAGCACATAATGGTTTGTG- ${ }^{\prime}$. The FGF1 primers were forward, 5'-CCAACCCAGGAGATCATTTG-3' and reverse, 5'-ACCCAGCCTGACAGACAATC- $3^{\prime}$. The FGF7 primers were forward, $5^{\prime}$-TGGGCACTATATCTCTAGCTTGC$3^{\prime}$ and reverse, 5'-GGGTGCGACAGAACAGTCT-3'. The FGF9 primers were forward, $5^{\prime}$-TCACTTGAGCCCTTAAAACATAT AAATGCTTTCATGCGGTG-3' and reverse, $5^{\prime}$-CACCGCAT GAAAGCATTTATATGTTTTAAGGGCTCAAGTG- ${ }^{\prime}$. The U6 primers were forward, 5'-CTCGCTTCGGCAGCA CA- $3^{\prime}$ and reverse, 5'-ACGCTTCACGAATTTGCGTGTC$3^{\prime}$. The GAPDH primers were forward, 5'-GATGAGTTCA GGCAACATC- $3^{\prime}$ and reverse, 5'-TGGTGAAGACGCCAG TGGA-3'.

\section{The Dual-Luciferase Reporter Assay}

The circRNA-Ep400 binding site and the FGF-1/7/9 $3^{\prime}$-UTR, termed circRNA-Ep400-WT, circRNA-Ep400-Mut, FGF-1/7/9$3^{\prime}$ UTR WT, and PROK2-3'-UTR-Mut were placed in a pGL3 promoter vector (Realgene, Nanjing, China) using $\mathrm{KpnI}$ and HindIII sites in a dual-luciferase reporter assay. 293T cells were plated into 24-well plates, and then transfected 5 ng Renilla of the luciferase vector, pRL-SV40, $50 \mathrm{nM}$ miR-15b-5p mimics, 80 ng plasmid, and negative control using Lipofectamine 2000 (Invitrogen). Cells were collected and assessed 2 days after transfection using a Dual-Luciferase Assay (Promega, Madison, WI, United States). The experiments were independently repeated three times.

\section{The 5-Ethynyl-2'-Deoxyuridine (EdU) Assay}

An EdU assay kit (RiboBio, Guangzhou, China) was used to measure DNA synthesis and cell proliferation. We seeded 10,000 NIH 3T3 cells and tenocytes, which were previously treated in a 96-well plate overnight. The following day, we added $25 \mu \mathrm{M}$ Edu solution to 96-well plate, followed by incubation and observation the next day. A solution of $4 \%$ formalin was then used to fix the cells for $2 \mathrm{~h}$ at room temperature. Next, $0.5 \%$ Triton X-100 was used to permeabilize the cells for $10 \mathrm{~min}$. Next, $200 \mu \mathrm{L}$ of the Apollo reaction solution was added for $30 \mathrm{~min}$ to stain for EdU, after which $200 \mu \mathrm{L}$ of DAPI was added to stain the nuclei. Finally, a light microscope (Nikon, Tokyo, Japan) was used to detect cell proliferation and DNA synthesis, which was reflected by blue and red signals, respectively.

\section{The Transwell and Wound Healing Assays}

Fibroblast and tenocyte cells were suspended in $200 \mu \mathrm{L}$ of serumfree medium and added to an upper Transwell chamber with $8 \mu \mathrm{m}$ pores (Corning Costar, Corning, NY, United States) We then added $600 \mu \mathrm{L}$ of medium, including $20 \%$ FBS, to the lower chamber as a chemoattractant. After incubation for 1 day, we immersed the cells in the filter with methanol, which were then stained with $0.1 \%$ Crystal Violet. Finally, the stained cells were counted in three fields of view $(200 \times)$.

\section{Statistical Analyses}

Differences between both groups were assessed by paired or unpaired two-tailed $t$-tests. Pearson's correlation test was used to determine associations between both groups. One-way ANOVA and the non-parametric with Kruskal-Wallis test were used for multiple groups comparisons. We expressed the results as the mean \pm SEM. We considered $p<0.05$ as significant. Prism software (GraphPad, San Diego, CA, United States) was used for all statistical analyses.

\section{RESULTS}

\section{Macrophages Play an Important Role After TI}

To identify the role of BMDMs in peritendinous fibrosis after TI, we depleted macrophages using Clo-Lipo 2 days before and just prior to tendon surgery (days 2 and 0 , respectively) and at 1,2 , and 3 weeks following TI, while the control group received PBS-containing liposomes. We allocated the mice into the three groups in a random manner involving the sham operation (NC), and FDL TI, and TI with clodronate liposomes (Clo-Lipo) treatment (TI-Clo). The results of the immunofluorescence detection showed that $\mathrm{F} 4 / 80$ expression was increased after TI, suggesting that macrophages had infiltrated into the damaged tissues. In contrast, Clo-Lipo pretreatment suppressed macrophage infiltration (Figure 1A). Masson staining showed that fibrogenesis was increased at 21 days after TI. Macrophage depletion (TI + Clo-Lipo group) decreased fibrogenesis, suggesting that macrophage depletion efficiently alleviated peritendinous fibrosis, revealing the role of macrophages in the development of tendon adhesion (Figure 1B). Western blot analysis showed that macrophage depletion inhibited peritendinous fibrosis by decreasing the accumulation of extracellular matrix (ECM) components such as 


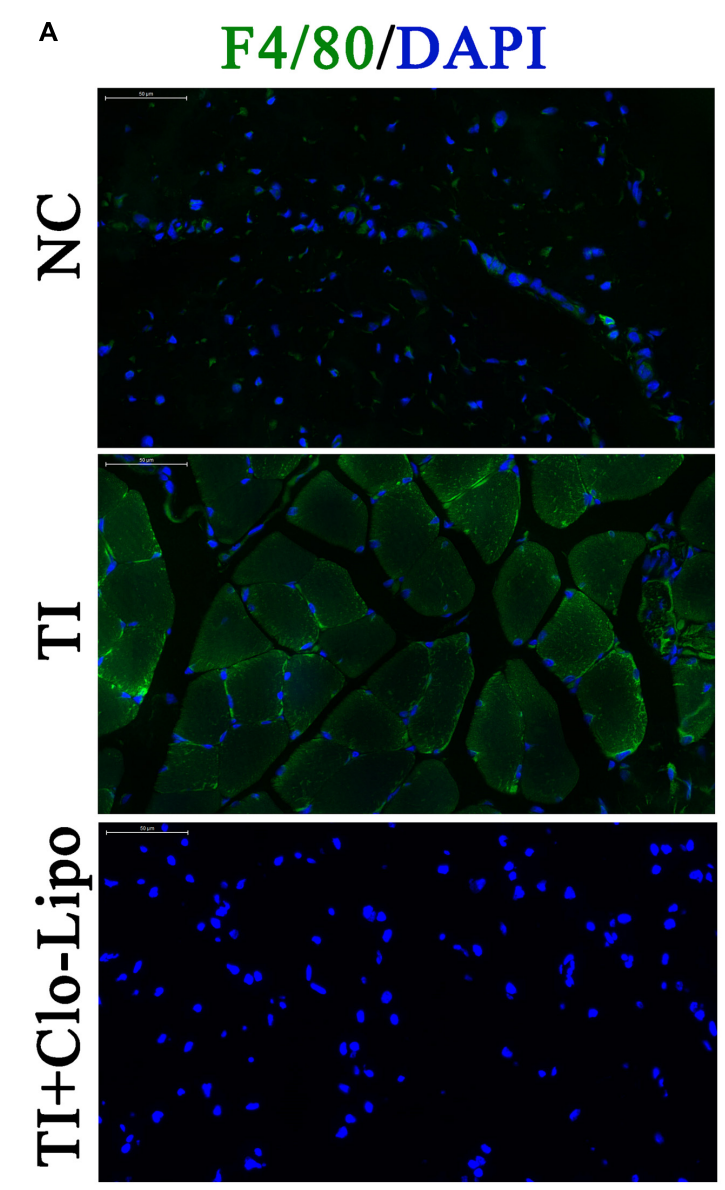

\section{B Masson staining}

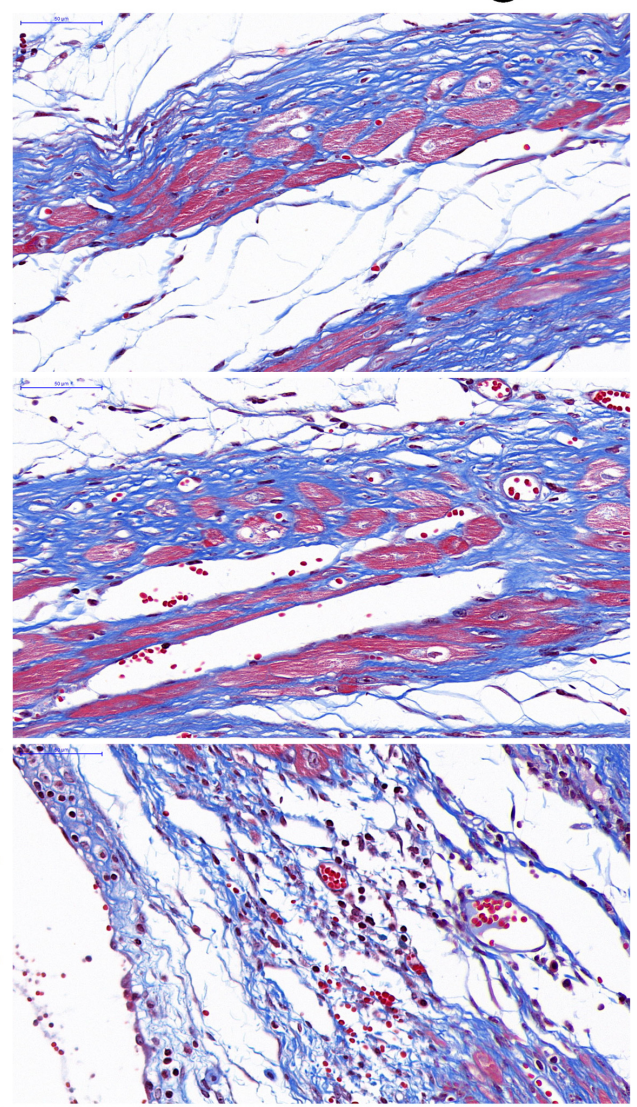

C
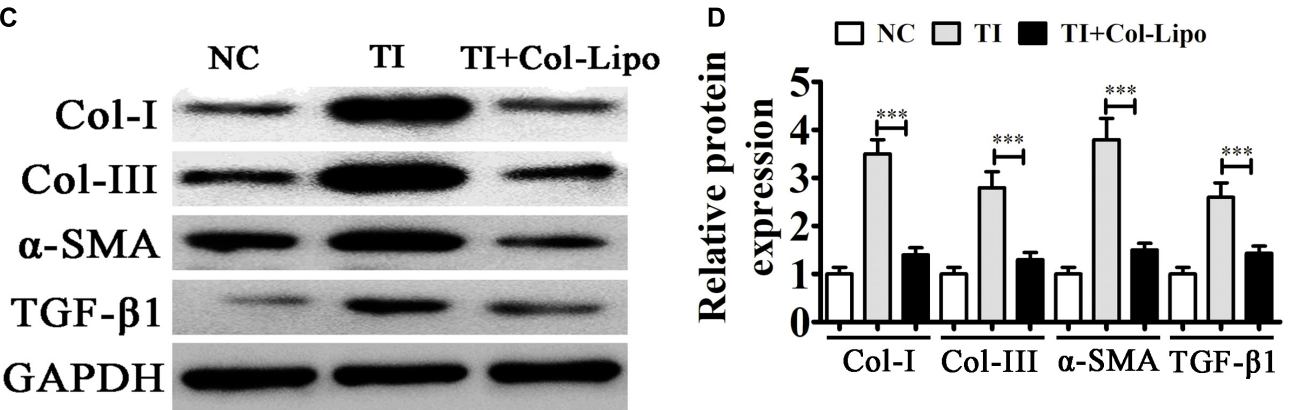

FIGURE 1 | Macrophages play an important role after tendon injury (TI) with or without Clo-containing liposomes. (A) Immunostaining of macrophages (F4/80 ${ }^{+}$cells were green) in each group; the cell nuclei were stained with 4,6-diamidino-2-phenylindole (blue). (B) Masson's trichrome staining showing the fibrosis of tendon tissue. (C,D) Western blot analysis and quantification of the Col I, Col III, and $\alpha$-SMA, as well as TGF- $\beta 1$ expression levels. The results are expressed as the mean $\pm \mathrm{SD},{ }^{* * *} P<0.001$ vs. Tl.

collagen type I (COL I), COL III, and alpha smooth muscle actin ( $\alpha$-SMA) in injured tendons, as well as TGF- $\beta 1$ (Figures 1C,D).

\section{Macrophage Phenotypic Transformation Plays a Role in Regulating Peritendinous Fibrosis After TI}

Our immunofluorescence study also showed that iNOS (M1 macrophage marker) expression during peritendinous was increased after TI (Figure 2A). The result also showed that M2 macrophages showed almost no difference between the NC and TI groups using CD206 staining (red) (Supplementary Figure 1). Other studies have reported that persistent tilt polarization toward M2 macrophages correlated with fibrosis and the epithelia-mesenchymal transition (Wang Y. Y. et al., 2017; Wang et al., 2019; Lu et al., 2018). To determine if M2 macrophages promoted peritendinous fibrosis posterior TI by secreting exosomes, IL-4 and IL-13 at $20 \mathrm{ng} / \mathrm{mL}$ was used for M2 

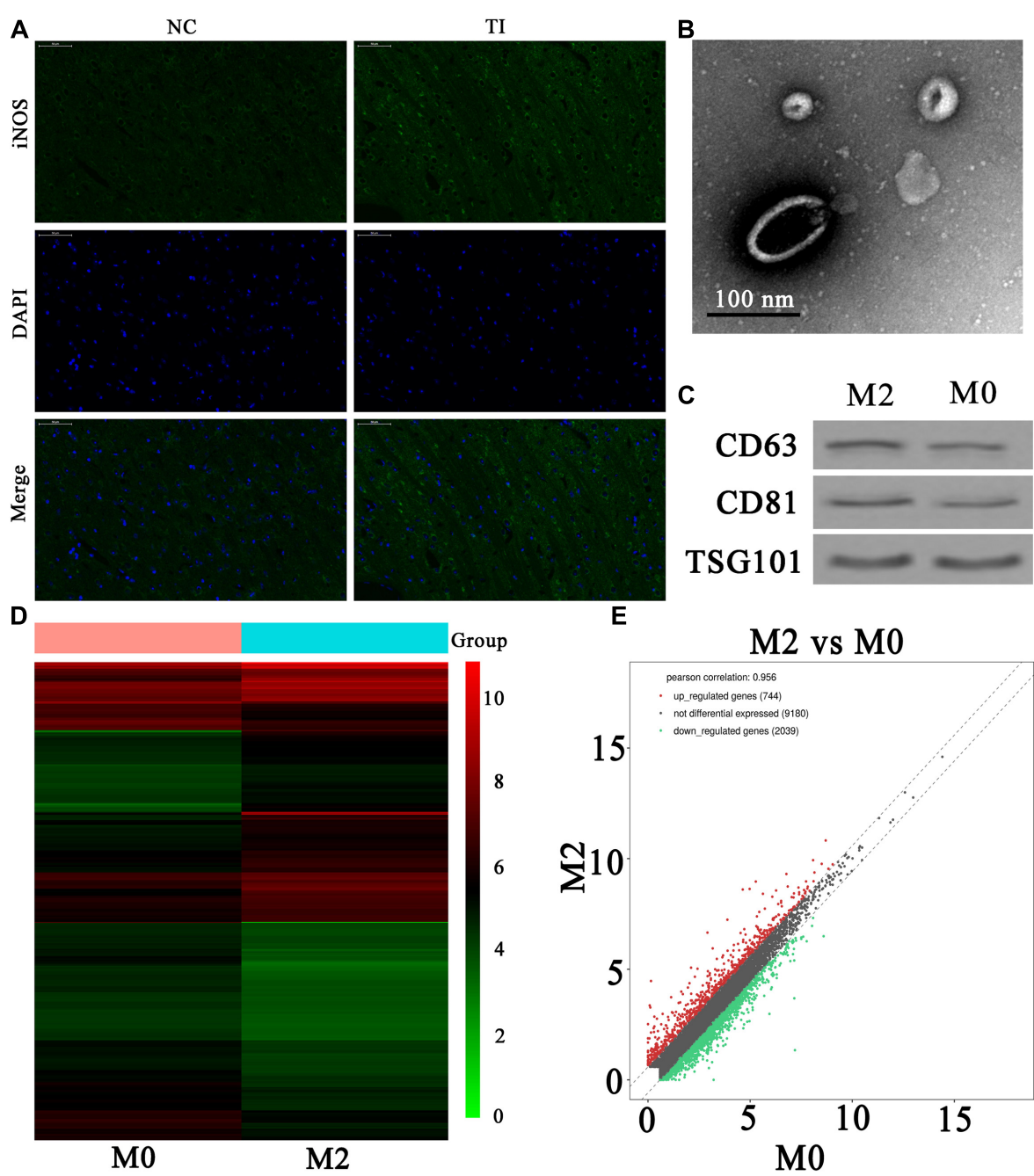

M2 vs M0

$\mathbf{F}$

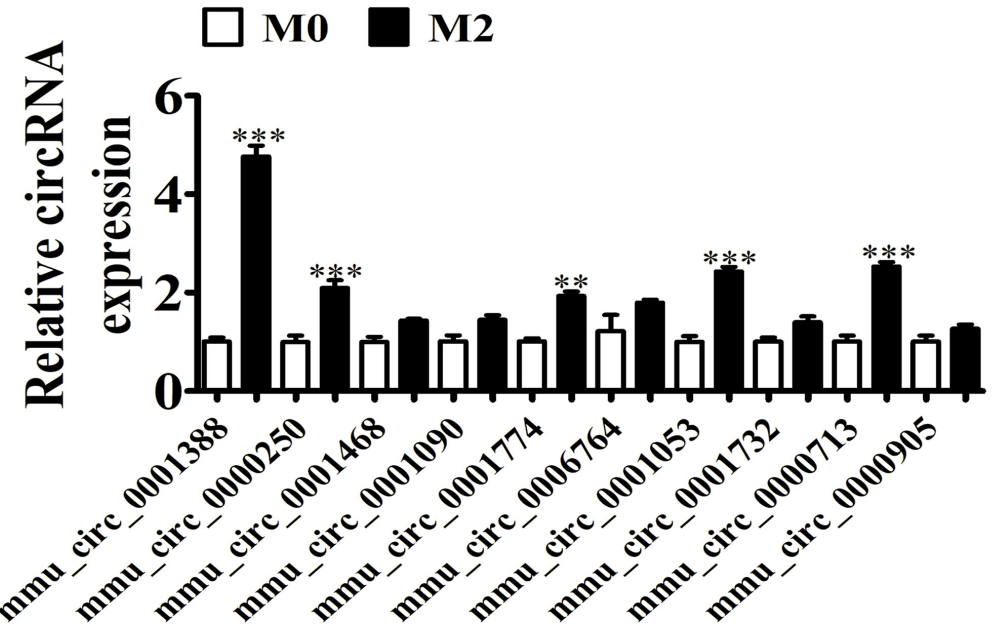

FIGURE 2 | Macrophage phenotypic transformation plays a role in regulating peritendinous fibrosis after tendon injury (TI). (A) Immunofluorescence detection showing the expression of iNOS (M1 marker). (B) Transmission electron microscopy shows exosome secretion from macrophages. (C) Western blot analysis showing the expression of exosome specificity markers (CD61, CD81, and TSG101). (D) A heat map shows the differentially expressed circRNAs. (E) A volcano plot showing the upregulation and downregulation of circRNA from M0 and M2 macrophage exosomes. (F) RT-qPCR detection comparing the upregulated expression of ten circRNAs from M0 and M2 macrophage exosomes. The results are expressed as the mean $\pm \mathrm{SD},{ }^{* *} P<0.01,{ }^{* * *} P<0.001 \mathrm{vs}$. M0 macrophages. 


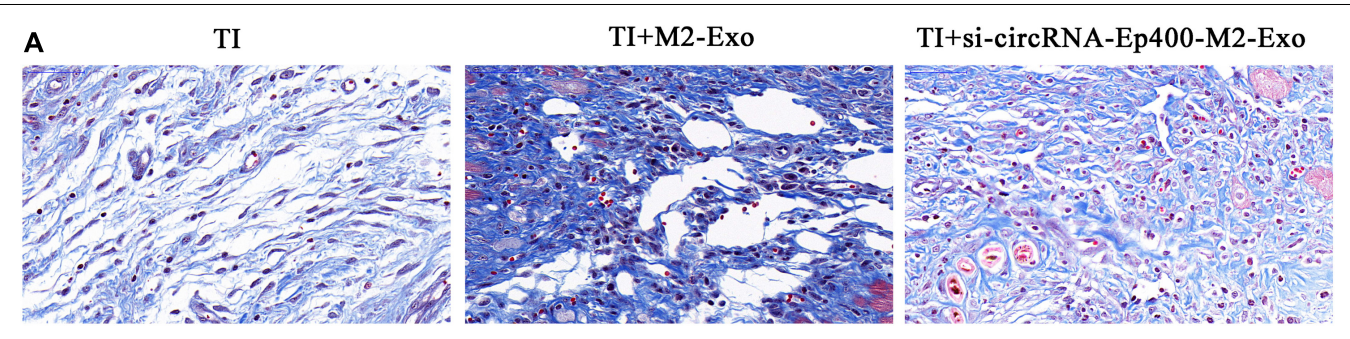

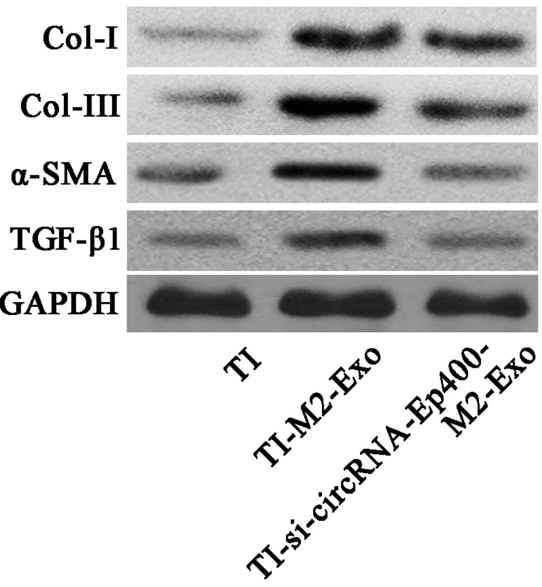

C $\square$ TI $\square$ TI+M2-Exo

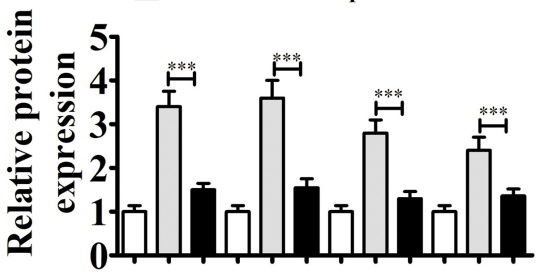

$\mathrm{c}^{2} \mathrm{cos}^{2}$ के
D

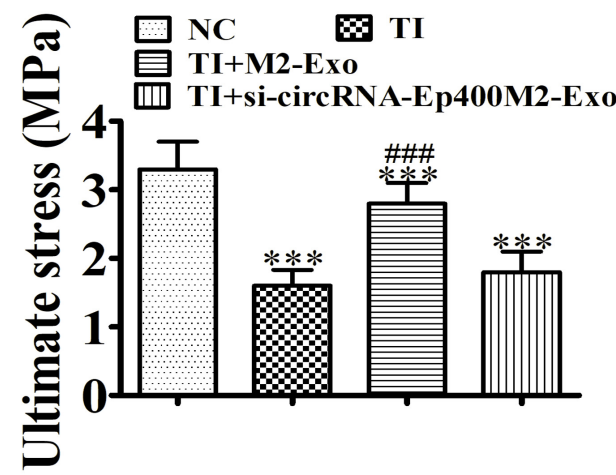

$\mathrm{N}$ NC $\mathrm{TI}$

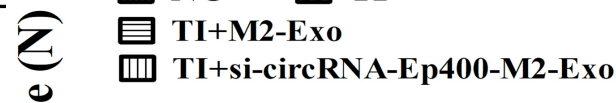

TI+si-circRNA-Ep400-M2-Exo
泀

G

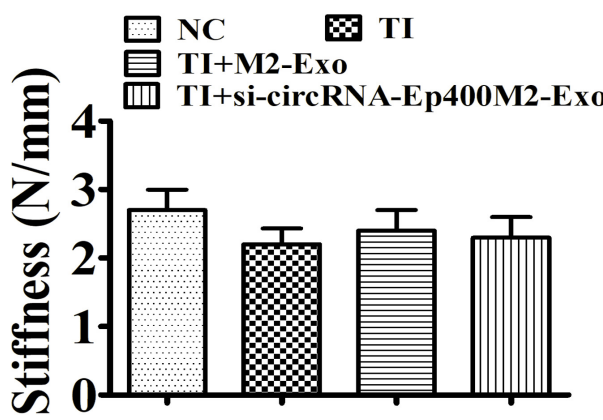

FIGURE 3 | M2 macrophage-derived circRNA-Ep400-containing exosomes function in promoting peritendinous fibrosis after tendon injury. (A) Masson's trichrome staining showing the fibrosis of tendon tissue. (B,C) Western blot analysis and quantification of the Col I, Col III, and $\alpha$-SMA, as well as TGF- $\beta 1$ expression levels. The results are expressed as the mean $\pm \mathrm{SD},{ }^{* * *} P<0.001$. (D-G) The tensile strengths of tendons after impairment were measured, including ultimate stress (D), ultimate load failure (E), Young's modulus (F), and stiffness (G). The results are expressed as the mean \pm SD. ${ }^{* * *} P<0.001$ vs. NC. \#\# $P<0.01, \# \# P<0.001$ vs. TI. 
macrophage induction. Electron microscopy results suggested abundant macrophage-derived exosomes having diameters of 70-150 nm (Figure 2B). Western blotting showed that the vesicles were positive for the exosome-specific markers of CD63, CD81, and TSG101 (Figure 2C), confirming that the structures were mainly exosomes, and that they were derived from macrophages.

To identify the regulatory mechanism, exosomes from M0 and M2 macrophages were used for high-throughput RNASeq. The result showed that there were 744 upregulated and 2,039 downregulated circRNAs in M2 macrophage exosomes, when compared with M1 macrophage exosomes (Figures 2D,E and Supplementary Figure 1). We selected ten upregulated circRNAs for RT-qPCR detection. The results showed that mmu_circ_0001388 (circRNA-Ep400) significantly increased (approximately fivefold) (Figure 2F), suggesting that circRNAEp400 played a role in M2 macrophage exosome-mediated promotion of peritendinous fibrosis following TI.

\section{M2 Macrophage-Derived circRNA-Ep400-Containing Exosomes Function in Promoting Peritendinous Fibrosis After TI}

Masson's staining showed that fibrogenesis was increased 21 days after TI and M2 macrophage exosome treatment. However, exosomes from circRNA-Ep400-downregualted M2 macrophages effectively alleviated peritendinous fibrosis (Figure 3A). Western blotting showed that circRNA-Ep400 depletion of M2 macrophage exosomes inhibited peritendinous

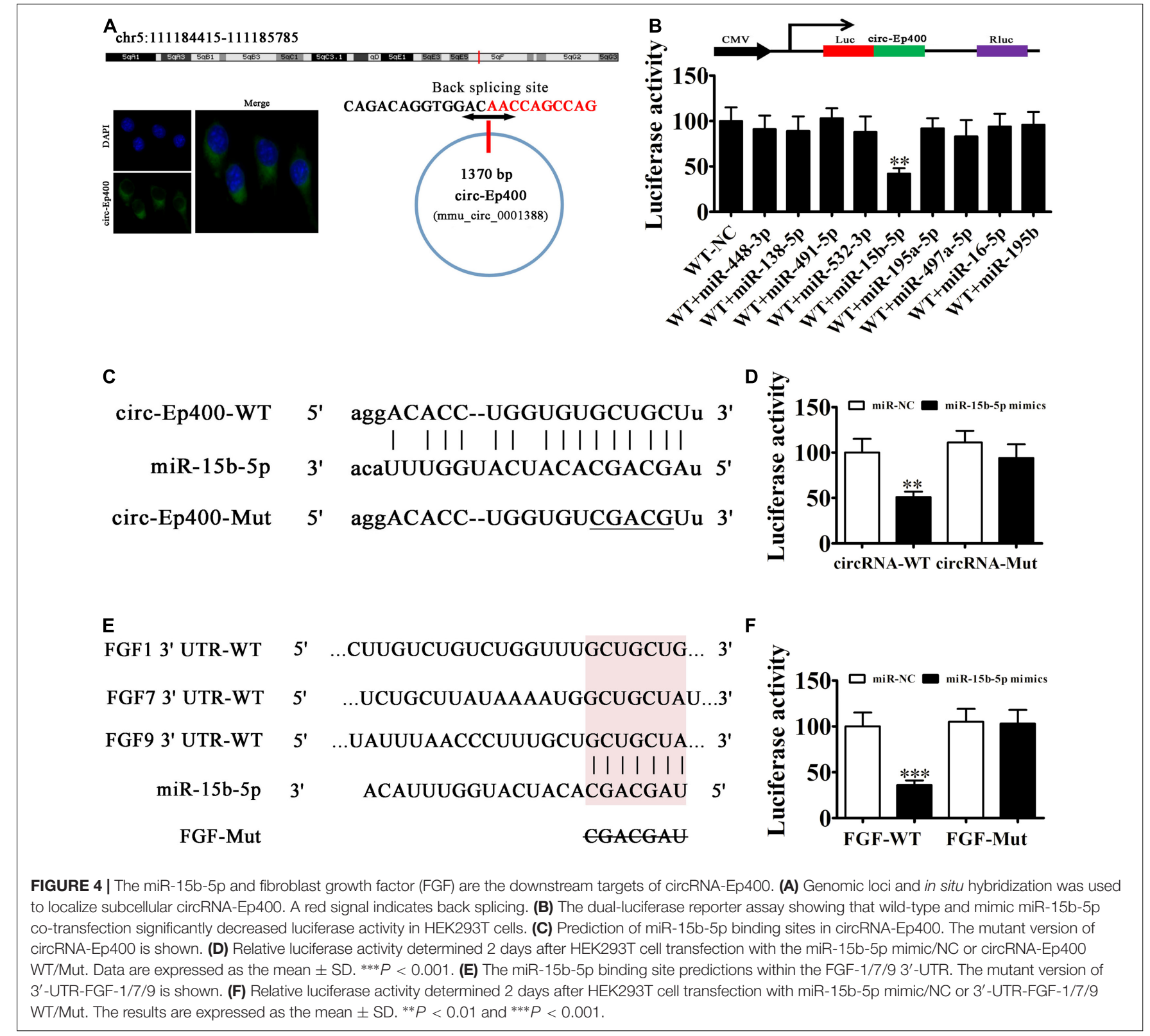


A

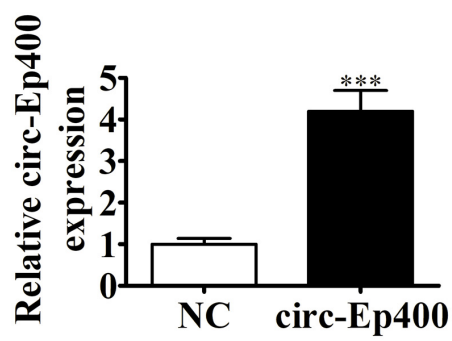

C

Fibroblast

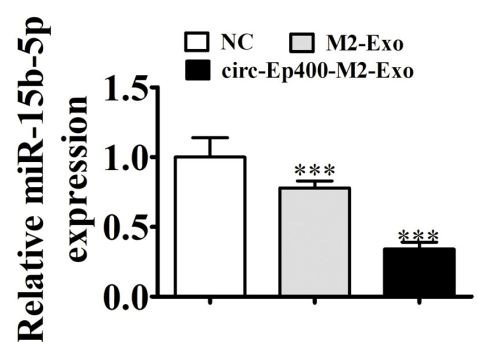

E Tendocytes

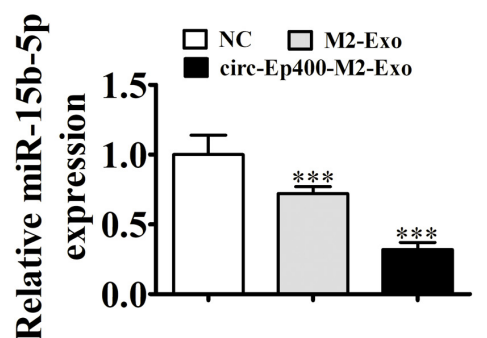

H

Fibroblast

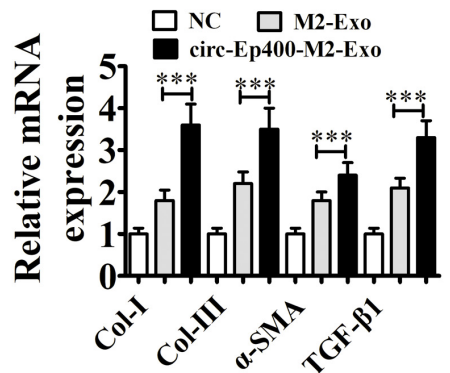

B

Fibroblast

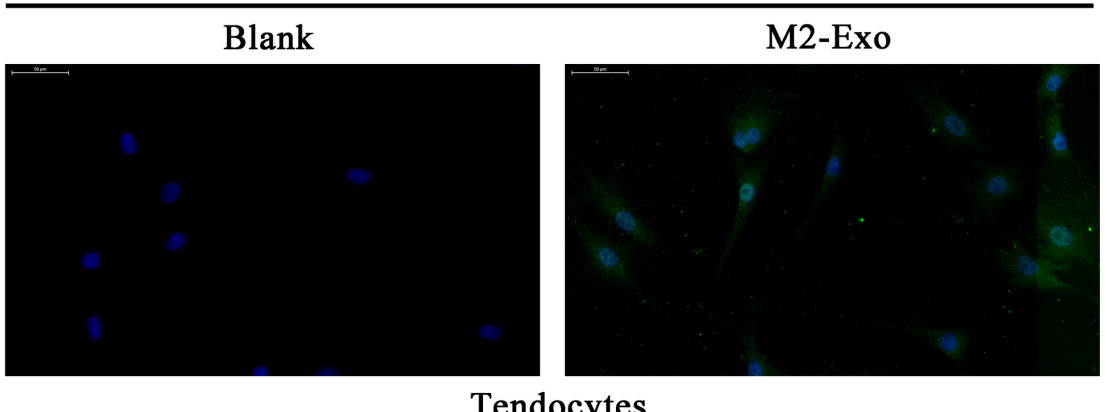

Tendocytes

D

Blank

M2-Exo
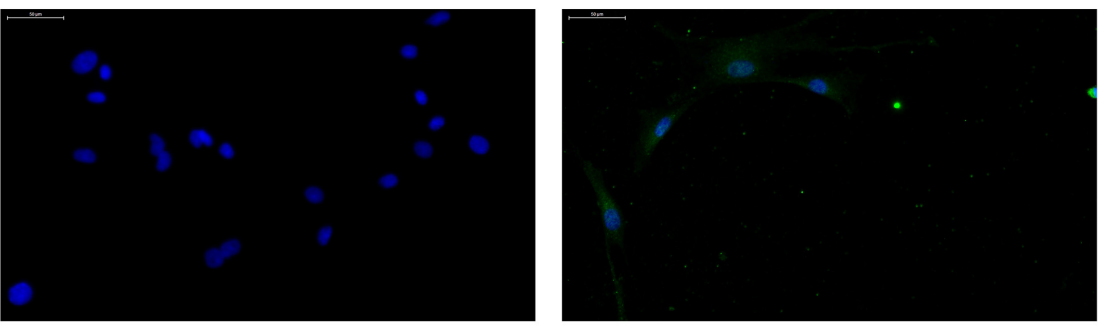

F Fibroblast

G Tendocytes
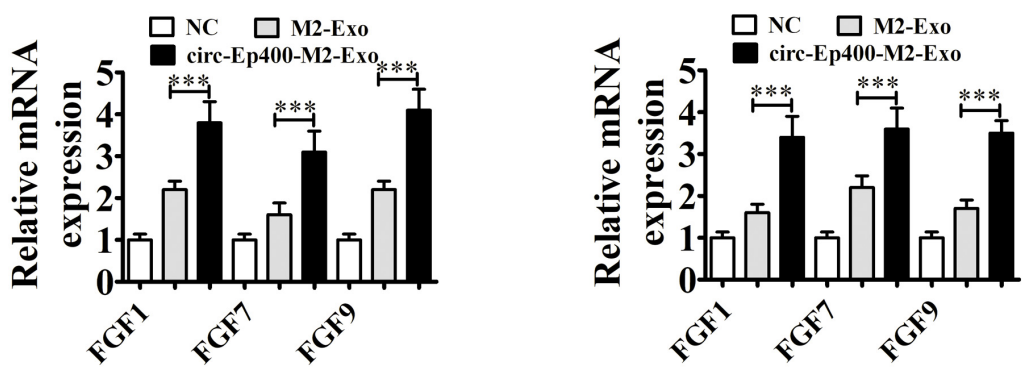

I Tendocytes

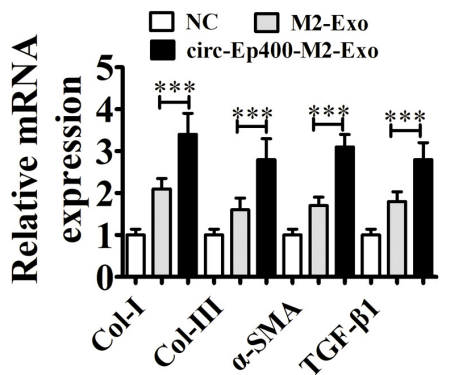

FIGURE 5 | M2 macrophage-derived circRNA-Ep400-containing exosomes promote profibrotic activity in tenocytes and fibroblasts in vitro. (A) RT-qPCR showing the circRNA-Ep400 expression in exosomes derived from M2 macrophages (NC) or overexpressed circRNA-Ep400 M2 macrophages. The results are expressed as the mean \pm SD. ${ }^{* * *} P<0.001$ vs. NC. (B) Fibroblasts were transfected with PKH26-labeled exosomes. (C) RT-qPCR showing the miR-15b-5p expression after treatment with exosomes from M2 macrophages or overexpressed circRNA-Ep400 M2 macrophages. The results are expressed as the mean \pm SD. ${ }^{* * *} P<0.001$ vs. the NC. (D) Tenocytes transfected with PKH26-labeled exosomes. (E) RT-qPCR showing miR-15b-5p expression after treatment with exosomes from M2 macrophages or overexpressed circRNA-Ep400 M2 macrophages. The results are expressed as the mean $\pm \mathrm{SD}$. ${ }^{* * *} P<0.001$ vs. the NC. (F,G) RT-qPCR showing FGF1, FGF-7, and FGF-9 expression in both fibroblasts and tenocytes after treatment with exosomes from M2 macrophages or overexpressed circRNA-Ep400 M2 macrophages. The results are expressed as the mean \pm SD. ${ }^{* * *} P<0.001$. (H,I) RT-GPCR showing the Col-I, Col-III, and $\alpha$-SMA, as well as TGF- $\beta 1$ expression levels in both tenocytes and fibroblasts after treatment with exosomes from M2 macrophages or overexpressed circRNA-Ep400 M2 macrophages. The results are expressed as the mean $\pm \mathrm{SD}$. ${ }^{* * *} P<0.001$. 
fibrosis by decreasing ECM component accumulations including Col-I, Col-III, and $\alpha$-SMA in injured tendons, as well as TGF$\beta 1$ (Figures 3B,C), suggesting that M2 macrophage-derived circRNA-Ep400-containing exosomes played an important role in the induction of peritendinous fibrosis as a result of TI. Biomechanical tests can be used to evaluate the tensile strength of tendons after impairment. The mean tensile strength of normal tendons was $34.32 \pm 2.24$. The tensile strength had decreased during 3 weeks. M2 macrophage exosome treatment increased the tensile strength of tendons, but after downregulation of circRNA-Ep400, the therapeutical effect was inhibited. The results also showed that M2 macrophage exosome treatment significantly increased ultimate stress and Young's modulus. No significant difference was found in stiffness (Figures 3D-G).

\section{miR-15b-5p and Fibroblast Growth Factor (FGF) Are the Downstream Targets of circRNA-Ep400}

CircRNA-Ep400 originates from circularizing exons from the Ep400 gene at chr5:111184415-111185785. Ep400 is comprised of 1,370 bps and the spliced mature circRNA is comprised of 1,370 bps (Figure 4A). FISH assays found that circRNAEp400 were predominantly localized to the cytoplasm. An increasing number of studies have confirmed that circRNAs, including miRNA/miR response elements, can connect miRNAs as competitive endogenous RNAs to regulate the expression of target mRNAs (Lin and Chen, 2018; Panda, 2018). Biological information analysis combined with luciferase reporter analysis has shown that circRNA-Ep400 cannot interact with miR-195a5p, miR-138-5p, miR-448-3p, miR-491-5p, miR-532-3p, miR497a-5p, miR-16-5p, and miR-195b. However, circRNA-Ep400 only interacted with miR-15b-5p, because miR-15b-5p inhibited luciferase activity in WT cells (Figure 4B). Bioinformatic analyses showed that miR-15b-5p was the downstream target of circRNAEp400. To establish a correlation between circRNA-Ep400 and miR-15b-5p, we constructed WT or mutant (MUT) circRNAEp400 sequences containing the miR-15b-5p binding sequence inserted into a luciferase reporter vector (Figure 4C). The luciferase reporter vector was then introduced into $293 \mathrm{~T}$ cells combined with or without the miR-15b-5p mimic. Luciferase reporter analysis showed that miR-15b-5p inhibited luciferase activity in WT cells, but not in MUT cell lines (Figure 4D), suggesting that miR-15b-5p was the target of circRNA-Ep400.

Bioinformatic analyses verified that FGF1/7/9 was the miR-15b-5p downstream target. To further characterize the correlations between miR-15b-5p and FGF1/7/9, we constructed WT or MUT 3 '-UTR-FGF1/7/9 sequences containing the miR15b-5p binding sequence inserted into the luciferase reporter vector (Figure 4E). The luciferase reporter vector was injected into $293 \mathrm{~T}$ cells combined with or without the miR-15b-5p mimic. The luciferase reporter analyses suggested that miR-15b$5 \mathrm{p}$ inhibited luciferase activity in WT cells, but not in MUT cell lines (Figure 4F), suggesting that FGF1/7/9 was the target of miR15b-5p.

\section{M2 Macrophage-Derived circRNA-Ep400-Containing Exosomes Promote Pro-fibrotic Activities of Tenocytes and Fibroblasts in vitro}

RT-qPCR detection showed that the expression of circRNAEp400 was significantly increased in exosomes derived from circRNA-Ep400 overexpressed M2 macrophages when compared with the NC group (Figure 5A). To determine if macrophagederived exosomes could be internalized by fibroblasts and tenocytes, we co-cultured fibroblasts and tenocytes with PKH26-labeled exosomes. Immunofluorescence detection showed that PKH26-labeled exosomes were tracked in both fibroblasts (Figure 5B) and tenocytes (Figure 5D). RT-qPCR analysis showed that miR-15b-5p expressions in both fibroblasts (Figure 5C) and tenocytes (Figure 5E) were decreased after treatment with exosomes from M2 macrophages, especially from circRNA-Ep400 overexpressed M2 macrophages. RT-qPCR analysis showed that the expressions of FGF1, FGF-7, and FGF-9 in both fibroblasts and tenocytes were increased after

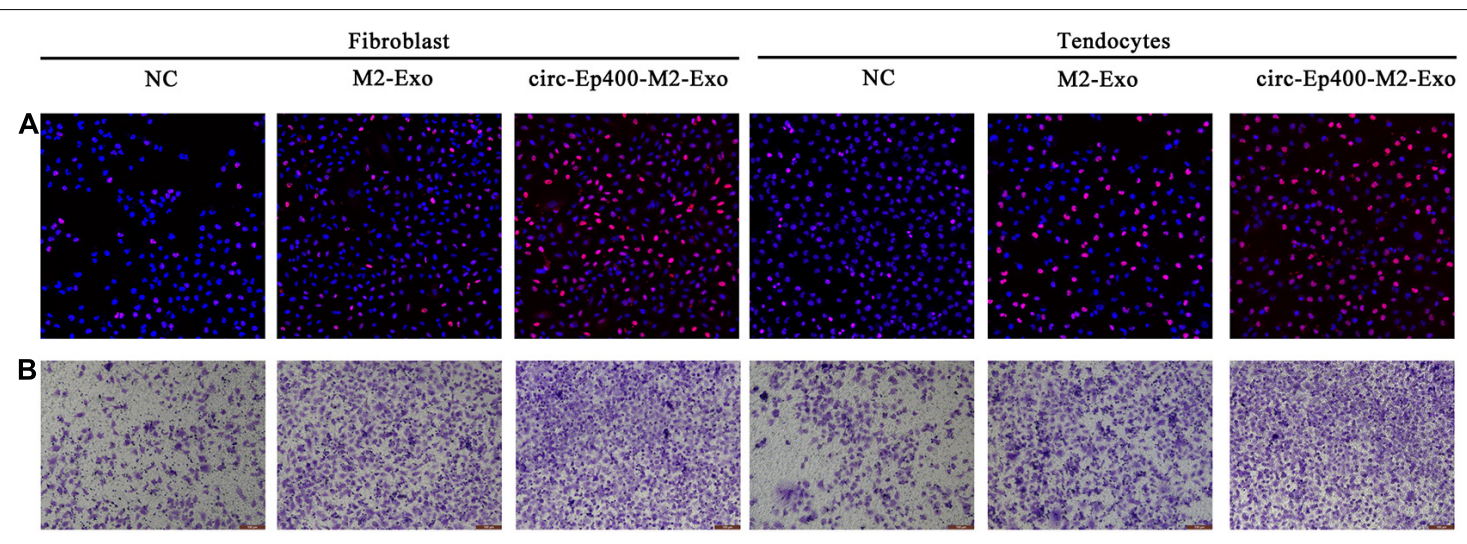

FIGURE 6 | M2 macrophage-derived circRNA-Ep400-containing exosomes promote proliferation and migration of fibroblasts and tenocytes in vitro. (A) The 5-ethynyl-2'-deoxyuridine assay showing the proliferative ability of both fibroblasts and tenocytes. (B) Transwell assays show the migration ability of both fibroblasts and tenocytes. 
treatment with exosomes from M2 macrophages, especially from circRNA-Ep400 overexpressed M2 macrophages (Figures 5F,G). The RT-qPCR detection also showed that Col-I, Col-III, and $\alpha$-SMA, as well as TGF- $\beta 1$, expressions in both tenocytes and fibroblasts were increased after treatment with exosomes from M2 macrophages, especially from overexpressed circRNA-Ep400 M2 macrophages (Figures $\mathbf{5 H}, \mathbf{I}$ ).

Edu (Figure 6A) and Transwell (Figure 6B) assays showed that the proliferation and migration ability were increased in both fibroblasts and tenocytes after treatment with exosomes from M2 macrophages, especially from overexpressed circRNAEp400 M2 macrophages.

\section{DISCUSSION}

In this study, we found that macrophages infiltrate in peritendinous tissues after TI. Macrophage depletion inhibited peritendinous fibrosis, suggesting that macrophages play an important regulatory role after TI. Immunohistochemical studies showed that the infiltrating macrophages were M1 macrophages. Due to the microenvironment, macrophages are categorized as alternatively activated M2 or typically activated M1 (Ji et al., 2016). M1 macrophages are induced form M0 macrophages by inflammatory stimuli, including lipopolysaccharides, and which are identified by high production of proinflammatory cytokines like IL-1 $\beta$, IL-12, reactive oxygen species, tumor necrosis factor$\alpha$, and iNOS, which suppress tissue recovery after aggravated injury (Benoit et al., 2008; Mege et al., 2011). The M2 phenotype, induced by the Th2 cytokines IL-13 or IL-4, are identified by arginase 1 production and detected in inflammatory zone 1 , and which are indispensable for inflammation resolution, tissue repair, and wound healing (Mantovani et al., 2004; Nair et al., 2006; Satoh et al., 2010). An increasing number of studies have found that exosomes secreted from macrophages play an important role in the regulation of the microenvironment (Bardi et al., 2018). Our study also characterized the regulatory role and mechanism of M2 macrophage exosomes after TI.

Our high-throughput sequencing found that circRNAs were abnormally expressed in exosomes from M0 and M2 macrophages. Further study showed that circRNA-Ep400 was highly expressed in M2 macrophage exosomes. CircRNA-Ep400 was localized in the cytoplasm and derived from circularizing exons from the Ep400 gene, which is located on chr5:111184415111185785. Previous studies have found that M2 macrophages are a major source of TGF- $\beta 1$ and platelet-derived growth factor, which, in turn, induce the differentiation of fibroblasts into myofibroblasts ( $\mathrm{Su}$ et al., 2015; Lipman et al., 2018). In this study we found that M2 macrophage exosome treatment promoted fibrosis by increased Col-I, Col-III, and $\alpha$-SMA, as well as TGF- $\beta 1$, expression after TI. Downregulation of circRNA-Ep400 suppressed the therapeutic effect (including tensile strength, ultimate stress, and Young's modulus of tendons) of M2 macrophage exosomes after TI.

Bioinformatics and luciferase report analyses confirmed that miR-15b-5p and FGF1/7/9 were downstream targets for circRNA-Ep400. High levels of circRNA-Ep400 containing exosome treatment inhibited miR-15b-5p, but promoted FGF1/7/9 expression in both fibroblasts and tenocytes.

An increasing number of studies have reported that FGF1/7/9 plays an important role in Achilles tendon restoration after TI (Reed and Johnson, 2014; Inoue et al., 2015; Guo et al., 2020). High circRNA-Ep400-containing exosome treatment promotes fibrosis by increasing Col-I, Col-III, and $\alpha$-SMA, as well as TGF- $\beta 1$, expression in both tenocytes and fibroblasts. High circRNA-Ep400 containing exosome treatment also promoted proliferation and migration in both fibroblasts and tenocytes after TI. Biomechanical tests found that M2 macrophage exosomes, especially high circRNA-Ep400containing exosome treatment, increased the tensile strength of tendons after impairment.

In conclusion, our study found that exosomes from M2 macrophage promoted peritendinous fibrosis posterior tendon injury via the miR-15b-5p/FGF-1/7/9 pathway by delivery of circRNA-Ep400. The study also confirmed a new mechanism responsible for macrophage-stromal cell communication, revealing a novel role for macrophage-derived exosomes in tendon adhesion, which could lead to new directions in clinical research.

\section{DATA AVAILABILITY STATEMENT}

The original contributions presented in the study are included in the article/Supplementary Material, further inquiries can be directed to the corresponding author/s.

\section{ETHICS STATEMENT}

The animal study was reviewed and approved by the Animal Research Committee in Shanghai Jiao Tong University Affiliated Shanghai First People's Hospital approved the animal protocols.

\section{AUTHOR CONTRIBUTIONS}

YY and BS conceived the study and wrote the manuscript with feedback from other authors. ZW, MY, ZC, and SL performed the experiments and their analyses. MJ and CY performed the experiments and contributed to the main text. All authors approved the final draft.

\section{FUNDING}

This investigation was supported by grants from Shanghai Pujiang Program (2019PJD045).

\section{SUPPLEMENTARY MATERIAL}

The Supplementary Material for this article can be found online at: https://www.frontiersin.org/articles/10.3389/fcell.2021. 595911/full\#supplementary-material 


\section{REFERENCES}

Bardi, G. T., Smith, M. A., and Hood, J. L. (2018). Melanoma exosomes promote mixed M1 and M2 macrophage polarization. Cytokine 105, 63-72. doi: 10.1016/ j.cyto.2018.02.002

Bartel, D. P. (2004). MicroRNAs: genomics, biogenesis, mechanism, and function. Cell 116, 281-297.

Benoit, M., Desnues, B., and Mege, J. L. (2008). Macrophage polarization in bacterial infections. J. Immunol. 181, 3733-3739. doi: 10.4049/jimmunol.181. 6.3733

Cui, H., He, Y., Chen, S., Zhang, D., Yu, Y., and Fan, C. (2019). MacrophageDerived miRNA-Containing Exosomes Induce Peritendinous Fibrosis after Tendon Injury through the miR-21-5p/Smad7 Pathway. Mol. Ther. Nucleic Acids 14, 114-130. doi: 10.1016/j.omtn.2018.11.006

de Jong, J. P., Nguyen, J. T., Sonnema, A. J., Nguyen, E. C., Amadio, P. C., and Moran, S. L. (2014). The incidence of acute traumatic tendon injuries in the hand and wrist: a 10-year population-based study. Clin. Orthop. Surg. 6, 196-202. doi: 10.4055/cios.2014.6.2.196

Guo, D., Li, H., Liu, Y., Yu, X., Zhang, X., Chu, W., et al. (2020). Fibroblast growth factor-2 promotes the function of tendon-derived stem cells in Achilles tendon restoration in an Achilles tendon injury rat model. Biochem. Biophys. Res. Commun. 521, 91-97. doi: 10.1016/j.bbrc.2019.10.082

Haider, N., Boscá, L., Zandbergen, H. R., Kovacic, J. C., Narula, N., GonzálezRamos, S., et al. (2019). Transition of Macrophages to Fibroblast-Like Cells in Healing Myocardial Infarction. J. Am. Coll. Cardiol. 74, 3124-3135. doi: 10.1016/j.jacc.2019.10.036

Hays, P. L., Kawamura, S., Deng, X. H., Dagher, E., Mithoefer, K., Ying, L., et al. (2008). The role of macrophages in early healing of a tendon graft in a bone tunnel. J. Bone Joint Surg. Am. 90, 565-579. doi: 10.2106/jbjs.f. 00531

Inoue, M., Nakajima, M., Oi, Y., Hojo, T., Itoi, M., and Kitakoji, H. (2015). The effect of electroacupuncture on tendon repair in a rat Achilles tendon rupture model. Acupunct. Med. 33, 58-64. doi: 10.1136/acupmed-2014-010 611

Ji, J., Shu, D., Zheng, M., Wang, J., Luo, C., Wang, Y., et al. (2016). Microbial metabolite butyrate facilitates M2 macrophage polarization and function. Sci. Rep. 6:24838.

Lichtnekert, J., Kawakami, T., Parks, W. C., and Duffield, J. S. (2013). Changes in macrophage phenotype as the immune response evolves. Curr. Opin. Pharmacol. 13, 555-564. doi: 10.1016/j.coph.2013.05.013

Lin, X., and Chen, Y. (2018). Identification of Potentially Functional CircRNAmiRNA-mRNA Regulatory Network in Hepatocellular Carcinoma by Integrated Microarray Analysis. Med. Sci. Monit. Basic Res. 24, 70-78. doi: $10.12659 / \mathrm{msmbr} .909737$

Lipman, K., Wang, C., Ting, K., Soo, C., and Zheng, Z. (2018). Tendinopathy: injury, repair, and current exploration. Drug Des. Devel. Ther. 12, 591-603. doi: $10.2147 /$ dddt.s154660

Lu, H., Wu, L., Liu, L., Ruan, Q., Zhang, X., Hong, W., et al. (2018). Quercetin ameliorates kidney injury and fibrosis by modulating M1/M2 macrophage polarization. Biochem. Pharmacol. 154, 203-212. doi: 10.1016/j.bcp.2018.05. 007

Mantovani, A., Sica, A., Sozzani, S., Allavena, P., Vecchi, A., and Locati, M. (2004). The chemokine system in diverse forms of macrophage activation and polarization. Trends Immunol. 25, 677-686. doi: 10.1016/j.it.2004.09. 015

McCormick, A., Charlton, J., and Fleming, D. (1995). Assessing health needs in primary care. Morbidity study from general practice provides another source of information. BMJ 310:1534.

Mege, J. L., Mehraj, V., and Capo, C. (2011). Macrophage polarization and bacterial infections. Curr. Opin. Infect. Dis. 24, 230-234. doi: 10.1097/qco. 0b013e328344b73e

Momen-Heravi, F., Bala, S., Bukong, T., and Szabo, G. (2014). Exosomemediated delivery of functionally active miRNA-155 inhibitor to macrophages. Nanomedicine 10, 1517-1527. doi: 10.1016/j.nano.2014. 03.014

Nair, M. G., Guild, K. J., and Artis, D. (2006). Novel effector molecules in type 2 inflammation: lessons drawn from helminth infection and allergy. J. Immunol. 177, 1393-1399. doi: 10.4049/jimmunol.177.3.1393

Panda, A. C. (2018). Circular RNAs Act as miRNA Sponges. Adv. Exp. Med. Biol. $6: 2018$.
Pitt, J. M., Kroemer, G., and Zitvogel, L. (2016). Extracellular vesicles: masters of intercellular communication and potential clinical interventions. J. Clin. Invest. 126, 1139-1143. doi: $10.1172 /$ jci87316

Reed, S. A., and Johnson, S. E. (2014). Expression of scleraxis and tenascin C in equine adipose and umbilical cord blood derived stem cells is dependent upon substrata and FGF supplementation. Cytotechnology 66, 27-35. doi: 10.1007/ s10616-012-9533-3

Satoh, T., Takeuchi, O., Vandenbon, A., Yasuda, K., Tanaka, Y., Kumagai, Y., et al. (2010). The Jmjd3-Irf4 axis regulates M2 macrophage polarization and host responses against helminth infection. Nat. Immunol. 11, 936-944. doi: 10.1038/ni.1920

Su, S., Zhao, Q., He, C., Huang, D., Liu, J., Chen, F., et al. (2015). miR-142-5p and miR-130a-3p are regulated by IL- 4 and IL-13 and control profibrogenic macrophage program. Nat. Commun. 6:8523.

Thomopoulos, S., Parks, W. C., Rifkin, D. B., and Derwin, K. A. (2015). Mechanisms of tendon injury and repair. J. Orthop. Res. 33, 832-839. doi: 10.1002/jor.22806

Thomou, T., Mori, M. A., Dreyfuss, J. M., Konishi, M., Sakaguchi, M., Wolfrum, C., et al. (2017). Adipose-derived circulating miRNAs regulate gene expression in other tissues. Nature 542, 450-455. doi: 10.1038/nature21365

Wang, C., Zhang, C., Liu, L., A, X., Chen, B., Li, Y., et al. (2017). MacrophageDerived mir-155-Containing Exosomes Suppress Fibroblast Proliferation and Promote Fibroblast Inflammation during Cardiac Injury. Mol. Ther. 25, 192204. doi: 10.1016/j.ymthe.2016.09.001

Wang, L., Zhang, Y., Zhang, N., Xia, J., Zhan, Q., and Wang, C. (2019). Potential role of M2 macrophage polarization in ventilator-induced lung fibrosis. Int. Immunopharmacol. 75:105795. doi: 10.1016/j.intimp.2019.105795

Wang, X., Xie, L., Crane, J., Zhen, G., Li, F., Yang, P., et al. (2018). Aberrant TGFbeta activation in bone tendon insertion induces enthesopathy-like disease. J. Clin. Invest. 128, 846-860. doi: 10.1172/jci96186

Wang, Y. Y., Jiang, H., Pan, J., Huang, X. R., Wang, Y. C., Huang, H. F., et al. (2017). Macrophage-to-Myofibroblast Transition Contributes to Interstitial Fibrosis in Chronic Renal Allograft Injury. J. Am. Soc. Nephrol. 28, 2053-2067. doi: 10.1681/asn.2016050573

Wynn, T. A., and Ramalingam, T. R. (2012). Mechanisms of fibrosis: therapeutic translation for fibrotic disease. Nat. Med. 18, 1028-1040. doi: 10.1038/nm.2807

Wynn, T. A., and Vannella, K. M. (2016). Macrophages in Tissue Repair, Regeneration, and Fibrosis. Immunity 44, 450-462. doi: 10.1016/j.immuni. 2016.02.015

Yao, Y., Wang, Y., Zhang, Z., He, L., Zhu, J., Zhang, M., et al. (2016). Chop Deficiency Protects Mice Against Bleomycin-induced Pulmonary Fibrosis by Attenuating M2 Macrophage Production. Mol. Ther. 24, 915-925. doi: 10.1038/ mt.2016.36

Ying, W., Riopel, M., Bandyopadhyay, G., Dong, Y., Birmingham, A., Seo, J. B., et al. (2017). Adipose Tissue Macrophage-Derived Exosomal miRNAs Can Modulate In Vivo and In Vitro Insulin Sensitivity. Cell 171, 372-384.e12.

Zhang, L., Zhang, S., Yao, J., Lowery, F. J., Zhang, Q., Huang, W. C., et al. (2015). Microenvironment-induced PTEN loss by exosomal microRNA primes brain metastasis outgrowth. Nature 527, 100-104. doi: 10.1038/nature15376

Zhou, Y. L., Yang, Q. Q., Yan, Y. Y., Zhu, C., Zhang, L., and Tang, J. B. (2018). Localized delivery of miRNAs targets cyclooxygenases and reduces flexor tendon adhesions. Acta Biomater. 70, 237-248. doi: 10.1016/j.actbio.2018.01. 047

Conflict of Interest: The authors declare that the research was conducted in the absence of any commercial or financial relationships that could be construed as a potential conflict of interest.

Publisher's Note: All claims expressed in this article are solely those of the authors and do not necessarily represent those of their affiliated organizations, or those of the publisher, the editors and the reviewers. Any product that may be evaluated in this article, or claim that may be made by its manufacturer, is not guaranteed or endorsed by the publisher.

Copyright (c) 2021 Yu, Sun, Wang, Yang, Cui, Lin, Jin and Yi. This is an open-access article distributed under the terms of the Creative Commons Attribution License (CC BY). The use, distribution or reproduction in other forums is permitted, provided the original author(s) and the copyright owner(s) are credited and that the original publication in this journal is cited, in accordance with accepted academic practice. No use, distribution or reproduction is permitted which does not comply with these terms. 